\title{
A Case of Wernicke Encephalopathy in a Dementia Caregiver: the Need for Nutritional Evaluation in Family Caregivers
}

Mayumi Ishida ( $\square$ mayumi_i@saitama-med.ac.jp )

Saitama Medical University International Medical Center https://orcid.org/0000-0003-1388-1284

Nozomu Uchida

Ogano Town Central Hospital

Kumi Itami

Saitama Medical University International Medical Center

Izumi Sato

Graduate School of Medicine and Public Health, Kyoto University

Akira Yoshioka

Mitsubishi Kyoto Hospital

Hideki Onishi

Saitama Medical University International Medical center

\section{Case report}

Keywords: Dementia, Caregiver, Thiamine defeciency, Wernickeencephalopathy

Posted Date: August 12th, 2020

DOl: https://doi.org/10.21203/rs.3.rs-56276/v1

License: (9) (i) This work is licensed under a Creative Commons Attribution 4.0 International License.

Read Full License

Version of Record: A version of this preprint was published at Journal of General and Family Medicine on September 12th, 2021. See the published version at https://doi.org/10.1002/jgf2.494. 
【The title of the paper 】

A case of Wernicke encephalopathy in a dementia caregiver: The need for nutritional evaluation in family caregivers

【The running title】

Thiamine deficiency in a caregiver

【The full names of all the authors, their affiliations】

Mayumi Ishida, C.P., Ph.D.1; Nozomu Uchida, M.D.2; Kumi Itami, C.N.S. 3; Izumi Sato, MPH, Ph.D.4; Akira Yoshioka, M.D., Ph.D. 5; and Hideki Onishi, M.D., Ph.D ${ }^{1}$.

1: Department of Psycho-oncology, Saitama Medical University International Medical Center, 1397-1 Yamane, Hidaka City, Saitama 350-1298, Japan

2: Department of General Medicine, Ogano Central Hospital, 300 Ogano, Ogano town, Saitama 368-0105, Japan

3: Department of Nursing, Saitama Medical University International Medical

Center, 1397-1 Yamane, Hidaka City, Saitama 350-1298, Japan 
4: Department of Pharmacoepidemiology, Graduate School of Medicine and Public Health, Kyoto University, Yoshida-honcho, Sakyo-ku, Kyoto city, Kyoto 606-8501, Japan

5: Department of Medical Oncology, Mitsubishi Kyoto Hospital, 1 Katsuragoosyo, Kyoto city, Kyoto 615-8087, Japan

【The name and e-mail address of the corresponding author】

Mayumi ISHIDA, C.P., Ph.D.

Associate Professor, Department of Psycho-oncology,

Saitama Medical University International Medical Center

1397-1 Yamane, Hidaka City, Saitama 350-1298, Japan

Tel. \& Fax: +81-42-984-4111

E-mail: mayumi_i@saitama-med.ac.jp

【Compliance with ethical standards】

Conflict of interest. The authors declare that they have no conflict of interest 


\section{Abstract}

Background:

We report a case in which a family member caring for her mother with dementia developed Wernicke encephalopathy, which is a neuropsychiatric disorder caused by acute/subacute thiamine deficiency, during the course of care.

Case presentation:

A 63-year-old woman consulted our psychiatric outpatient clinic complaining of difficulty in providing care. She had started caring for her mother with dementia 6 months previously, during which time she began to feel tired. In addition, a loss of appetite had appeared 2 months prior to her visit, and this had decreased to about $30 \%$ of normal from 10 days previously. Neurologically, she experienced mild unsteadiness, but she was fully conscious and had no ocular symptoms. Based on the fact that the store of thiamine in the body is exhausted in about 18 days, the possibility of thiamine deficiency was considered, and her unsteadiness disappeared after an intravenous injection of thiamine. Test results showed her serum thiamine level to be abnormally low, and the patient was diagnosed with Wernicke encephalopathy. 
Conclusions:

The burden of caring for a dementia patient may affect the nutritional status of the family caregiver. Thiamine deficiency should be one of the items considered as a nutritional issue in such caregivers.

\section{【Key words】}

Dementia, caregiver, thiamine deficiency, Wernicke encephalopathy 
A case of Wernicke encephalopathy in a dementia caregiver: The need for nutritional evaluation in family caregivers

\section{Background}

Dementia care is often provided by family members, and the responsibility for such care mainly falls to the spouse, children, or those familiar to the patient. The provision of care is a constant stressor that causes both physical and mental stress in the family caregiver [1]. However, the nutritional status of the caregiver, particularly issues related to vitamins, has not been discussed.

Thiamine, in its biologically active form thiamine pyrophosphate, is an essential coenzyme for oxidative metabolism [2]. As the human body cannot synthesize thiamine internally, thiamine must be ingested from food. The limit of thiamine storage in the human body is only 18 days [3]. Therefore, the body can become thiamine deficient through a loss of appetite lasting for a few weeks. A continuing thiamine deficiency can lead to the neuropsychiatric disorder Wernicke encephalopathy (WE) [4]. The classical symptoms of WE are delirium, ataxia and opthalmoplegia. This disorder is 
reversible if properly diagnosed and treated with parenteral thiamine administration. If left untreated, however, it causes severe and irreversible brain damage (Korsakoff syndrome) leading to death. The estimated mortality rate is about $20 \%$ [4]. Thiamine deficiency (TD) is recognized in various kinds of disease with associated loss of appetite including anorexia nervosa [5], diabetes mellitus [6], cancer [7, 8] and dementia [9]. However, no cases have been observed in family members caring for patients with dementia.

Here, we report a case in which a family member caring for her mother with dementia developed WE during the course of care. 


\section{Case presentation}

A 63-year-old woman consulted our psychiatric outpatient clinic complaining of difficulty in providing care.

Six months prior to her visit she had started living at home with her mother due to her mother being diagnosed with vascular dementia. However, coping with the symptoms of dementia in her mother resulted in considerable fatigue. For this reason, she determined that it was difficult to continue care at home and she sent her mother to a nursing home two months prior to her visit. However, even after her mother was in the nursing home, she frequently requested her daughter visit her, and during that time the caregiver came to feel even more physically fatigued.

The caregiver's appetite began to decline when she moved her mother into the nursing home, dropping to about $70 \%$ of normal and then to about $30 \% 10$ days before her visit to our clinic.

Physical examination at the time of consultation revealed general malaise. Neurologically, she experienced mild unsteadiness, but she was fully conscious and had no ocular symptoms. MRI examination of her head did not reveal any notable findings. Psychiatrically, neither depression nor a loss of 
motivation was observed.

Her medical history revealed she was diagnosed with breast cancer eight years previously, for which she was treated by surgery and chemotherapy. At the time of her consultation she was not receiving any anti-cancer drugs, and follow-up had not revealed any signs of recurrence. She had been diagnosed with depression nine years earlier. She was still visiting a psychiatric department, but her mental state was stable due to medication and psychotherapy. She had no history of alcohol or drug dependence.

Based on the findings that her appetite had begun to decline from 2 months previously and had dropped to only about $30 \%$ of normal from 10 days previously, the fact that thiamine stores in the body are depleted in about 18 days [3] as well as reports that WE can present with an absence of consciousness disorders, but only some unsteadiness [4, 10], we considered that this patient may have been suffering thiamine deficiency, and thiamine $100 \mathrm{mg}$ in combination with other B-group vitamins was administered twice intravenously, resolving her unsteadiness. Her serum thiamine level, as measured using high-performance liquid chromatography, was abnormally low at $21 \mathrm{ng} / \mathrm{ml}$ (reference range: $24-66 \mathrm{ng} / \mathrm{ml}$ ). Her vitamin B12 was 206 
$\mathrm{pg} / \mathrm{mL}$ (reference range: 180-914 $\mathrm{pg} / \mathrm{mL}$ ) and folic acid was $8.3 \mathrm{ng} / \mathrm{mL}$ (reference range: 4.00-999999.00 $\mathrm{ng} / \mathrm{mL}$ ), both of which were within the normal range. Further, no other blood biochemical abnormalities were found to explain the above-mentioned pathological condition. Based on these findings, she was diagnosed with WE. Thereafter, she experienced no further episodes of unsteadiness of gait. Oral administration of thiamine $75 \mathrm{mg}$ was continued, and her standard of daily living returned to the same level as before she began caring for her mother. 


\section{Discussion and Conclusions}

We experienced a case of WE in a family member caring for a dementia patient. This is the first reported case of WE resulting from thiamine deficiency due to the burden of dementia care.

Thiamine deficiency can be problematic in individuals with dementia [2, 9]. However, the nutritional status of the family members caring for a dementia patient has not been discussed to date. As WE may develop due to thiamine deficiency such as in this case, it may be necessary to consider the nutritional status, including thiamine, of family members caring for patients with dementia in the future.

The symptoms of WE are nonspecific and it is often difficult to diagnose. In particular, when there is no characteristic background factor such as heavy alcohol consumption as in this example, it becomes difficult to notice clinically. Moreover, in the case of a caregiver, clinical awareness may be delayed. In this case, WE diagnosis was triggered by a loss of appetite and unsteadiness. In this case, the fact that thiamine stores in the body are depleted in as little as 18 days [3] was beneficial to our diagnosis. This indicates that nutritional evaluation is important in making a clinical diagnosis in daily clinical practice. 
The caregiver in this case had a history of depression, and recurrence of depression was important in terms of differential diagnosis. However, in this case, the feeling of fatigue and loss of appetite reported by the patient corresponded to the diagnostic criteria of depression but did not meet the diagnostic criteria for depression, and it wasn't considered that they were caused by the deterioration in mental symptoms such as a recurrence of depression. In addition, as severe fatigue has been reported as a symptom of thiamine deficiency [11], the fatigue in this case was not considered to represent the deterioration in depression.

Although a characteristic symptom of WE is delirium, no delirium was observed in this case. However, as some cases of WE have been reported without delirium $[10,12]$, it is necessary to differentiate WE from other conditions even when there is no delirium observed as the continued oversight of WE results in Korsakoff syndrome with severe and irreversible brain damage.

In conclusion, this report emphasized the fact that the burden of caring for a dementia patient affects the nutritional status of the caregiver. Thiamine deficiency should be one of the items considered as a nutritional issue in such 
caregivers. Future research is expected to further clarify the nutritional issues in family members caring for people with dementia. 


\section{List of abbreviations}

WE: Wernicke encephalopathy

TD: Thiamine deficiency

\section{Declarations}

Ethics approval and consent to participate

Not applicable.

\section{Consent for publication}

Written consent was obtained from the patient.

\section{Availability of data and materials}

Not applicable.

\section{Competing interests}

The authors declare that they have no competing interests.

\section{Funding}

Not applicable.

\section{Authors' contributions}

IM and $\mathrm{OH}$ diagnosed the patient with Wernicke encephalopathy. IM prepared the manuscript and conducted literature research. $\mathrm{OH}$ added clinical information from a perspective of a psychiatrist, conducted additional literature research, edited the manuscript, and deepened discussion. NU, KI, IS, AY helped to deepen discussion on the unusual presentation of this case. All authors read and approved the final manuscript.

\section{Acknowledgements}

Not applicable. 


\section{References}

1. George LK, Gwyther LP. Caregiver well-being: a multidimensional examination of family caregivers of demented adults. Gerontologist 1986;26:253-9 doi:https://doi.org/10.1093/geront/26.3.253.

2. Sechi G, Sechi E, Fois C, Kumar N. Advances in clinical determinants and neurological manifestations of B vitamin deficiency in adults. Nutr Rev 2016;74:281300 doi:https://doi.org/10.1093/nutrit/nuv107.

3. MacLean LD, Rhode BM, Shizgal HM. Nutrition following gastric operations for morbid obesity. Ann Surg 1983;198:347-55.

4. Sechi G, Serra A. Wernicke's encephalopathy: new clinical settings and recent advances in diagnosis and management. Lancet Neurol 2007;6:442-55 doi:https://doi.org/S1474-4422(07)70104-7 [pii]

10.1016/S1474-4422(07)70104-7 [doi].

5. Winston AP, Jamieson CP, Madira W, Gatward NM, Palmer RL. Prevalence of thiamin deficiency in anorexia nervosa. Int $J$ Eat Disord 2000;28:451-4 doi:https://doi.org/10.1002/1098-108X(200012)28:4<451::AID-EAT14>3.0.CO;2-I [pii].

6. Thornalley PJ, Babaei-Jadidi R, Al Ali H, Rabbani N, Antonysunil A, Larkin J, Ahmed A, Rayman G, Bodmer CW. High prevalence of low plasma thiamine concentration in diabetes linked to a marker of vascular disease. Diabetologia 2007;50:2164-70 doi:https://doi.org/10.1007/s00125-007-0771-4 [doi].

7. Isenberg-Grzeda E, Rahane S, DeRosa AP, Ellis J, Nicolson SE. Wernicke-Korsakoff syndrome in patients with cancer: a systematic review. Lancet Oncol 2016;17:e142e8 doi:https://doi.org/10.1016/s1470-2045(16)00037-1.

8. Barbato M, Rodriguez PJ. Thiamine deficiency in patients admitted to a palliative care unit. Palliat Med 1994;8:320-4 doi:https://doi.org/10.1177/026921639400800408.

9. Uchida N, Ishida M, Sato I, Takahashi T, Furuya D, Ebihara Y, Ito H, Yoshioka A, Onishi H. Exacerbation of psychotic symptoms as clinical presentation of Wernicke Encephalopathy in an Alzheimer's disease patient. Journal of General and Family Medicine 2020;2020 doi:https://doi.org/10.1002/jgf2.330.

10. Onishi H, Ishida M, Tanahashi I, Takahashi T, Taji Y, Ikebuchi K, Furuya D, Akechi T. Wernicke encephalopathy without delirium in patients with cancer. Palliat Support Care 2018;16:118-21 doi:https://doi.org/10.1017/s1478951517000360.

11. Onishi H, Okabe T, Uchida N, Shirotake S, Todo M, Oyama M, Ishida M. Thiamine deficiency in a patient with recurrent renal cell carcinoma who developed weight loss with normal appetite and loss of energy soon after nivolumab treatment. Palliat 
Support Care 2020;18:241-3 doi:https://doi.org/10.1017/s1478951519000658.

12. Isenberg-Grzeda E, Shen MJ, Alici Y, Wills J, Nelson C, Breitbart W. High rate of thiamine deficiency among inpatients with cancer referred for psychiatric consultation: results of a single site prevalence study. Psychooncology 2017;26:13849 doi:https://doi.org/10.1002/pon.4155. 